Review

\title{
Microbial Transglutaminase is Beneficial to Food Industries but a Caveat to Public Health
}

Aaron Lerner ${ }^{1,2, *}$, Torsten Matthias ${ }^{2}$

1 B. Rappaport School of Medicine, Technion-Israel Institute of Technology, Haifa, 31096, Israel

2 AESKU.KIPP Institute, Wendelsheim, 55234, Germany

* Correspondence: Aaron Lerner, Email: aaronlerner1948@gmail.com; Tel.: +49-6734-9622-1010; Fax: +49-6734-9622-2222.

\begin{abstract}
Microbial transglutaminase (mTG) is a survival factor for bacteria that is heavily used as a protein glue in the processed food industries. Despite the manufacturers' claims for it safe usage, scientific observations are accumulating for its unwanted effects on human health. The enzyme can cross link proteins, imitating its family member, tissue transglutaminase, the autoantigen of celiac disease. Its gliadin cross-linked complexes are immunogenic in celiac disease. In the intestinal lumen, mTG exerts anti protease activity and forms resistant isopeptide bonds, it is anti-phagocytic, thus suppressing luminal protective pathways. It increases intestinal permeability, is trans-epithelialy transported and faces the enteric mucosal immune cells. Finally, mTG-containing products can react as emulsifiers and mucolytic agents thus compromising barriers' integrities. The present review summarizes and updates on the potential detrimental effects of mTG, aiming to protect the public from the enzyme's unwanted effects.
\end{abstract}

KEYWORDS: microbial transglutaminase; processed food; food additive; food industry; celiac disease; public health

\section{G Open Access}

Received: 30 November 2018 Accepted: 21 December 2018 Published: 04 January 2019

Copyright (c) 2019 by the author(s). Licensee Hapres, London, United Kingdom. This is an open access article distributed under the terms and conditions of Creative Commons Attribution 4.0 International License.

\section{INTRODUCTION}

Transglutaminases (TGs) (EC 2.3.2.13), i.e., protein-glutamine $\gamma$-glutamyltransferase, are multi-functional, pleiotropic enzymes, expressed ubiquitously and extensively in living organisms. They are active in all mammalian tissues, in invertebrates, plants, yeasts and bacterial cells. Presently, nine members of the TG family have been characterized in human tissues, playing a crucial role in physiological homeostasis, as well as in pathological disorders [1]. All TGs, catalyze the formation of an isopeptide bond, cross-linking an amine group (acyl acceptor) and the acyl group (acyl donor). They are an example of enzyme's induced posttranslational modification of proteins/peptides, involving a plethora of common chronic human diseases [2,3]. Deamidation or cross-linking of proteins are the main mechanisms through which they exert their biological functions. The human tissue 
tranglutaminase (tTG) is celebrating its diamond anniversary, since its discovery 60 years ago. It evolved from a pedestrian protein to a talented promising therapeutic target $[4,5]$.

The present review will concentrate on the prokaryotic TGs, the microbial TG (mTG), highlighting its massive uses in the processed food industries, as a food additive. It appears that mTG, a bacterial survival factor, is beneficial for the food industry, but, its public safety is under investigation where various potential detrimental effects were recently described or suggested.

\section{Characteristics of Microbial Transglutaminase}

Prokaryotic mTG is a member of the extended TG family, exerting deamidation and cross-linking of multiple substrates [6]. Its versatility spans a wide bacterial kingdom, was firstly isolated and characterized in Streptomyces mobaraense [2,3,7-9]. It is 331 amino acids long with a molecular weight of $37.9 \mathrm{kDa}$. Since its first characterization, a plethora of additional microbes were described to secrete $\mathrm{mTG}$, with variable enzymatic yield capacities [3]. The most frequently used industrial mTG is secreted from Streptoverticillium mobaraense [3]. Novel mTGs are continuously described using ultrahigh-throughput screening and other biotechnological systems [10]. A recent new one, for example that exerts anti-phagocytic activity was reported in Streptococcus suis [11].

\section{mTG and tTG are Structurally Different but Functionally Similar}

Inversely to the human tTG, considered as the autoantigen in celiac disease (CD) [12], mTG is not dependent on calcium for activation nor on nucleotides for deactivation. Instead of four domains, it has only a single structural domain and a lower molecular weight than the tTG. It exhibits less substrate specificity and operates in a wider $\mathrm{pH}$ range $[6,13]$. Bonds created by the $\mathrm{mTG}$ are relatively resistant to proteases degradation, the enzyme operates at a higher reaction rate, delivering a higher transamidation/deamidation ratio due to its improved cross-linking capacity. Notably, tTG is endogenous, while mTG is exogenous, a common enzyme of the prokaryotic kingdom, considered as an environmental factor that potentially can affect human health, as detailed below. Considering its protein modifying abilities, exerting deamidation and transamidation, it imitates functionally the endogenous tTG [2,3,6,13]. Based on its fundamental features and its wider enzymatic activity, it represent a prime candidate, extensively used by multiple and constantly developing industries. The tissue engineering, textile, leather, biomedical diagnostic, labeling, biotechnological, pharmaceutical, food processing and nutraceutical industries [6,8,14-22]. 


\section{Applications of Microbial Transglutaminase in the Food Processing Industries}

The industrial applications of mTG is constantly expanding and diverging. Since the present review zooms on human health and mTG ingestion, from now on it will focus on the food processing industries.

The surge in eight food additives, increasingly used by the processed food industries, mTG included, was summarized recently [14]. It appears that the net percent increase per year of total enzyme usage in the processed food industries is estimated to be $21.9 \%$, mTG being a major one [14]. It is estimated that for each kilogram of food material, the processed food industry is using $50-100 \mathrm{mg}$ of the mTG enzyme, ending up in a $15 \mathrm{mg}$ daily intake $[15,18,23]$.

The frequently used nickname of $\mathrm{mTG}$ is "protein glue" but scientifically, it is a clear posttranslational modifier of proteins. This results in a three-dimensional structural change consequently creating new epitopes on the complexes' surface [2,3,6,13]. In fact, the enzyme is consumed by most of the processed food industries, including bakeries, dairy, meat, surimi, sea food and fish, salad, casein and gelatin, myosin and actin, confection, convenience and many more industrial food applications [6,8,14-21,24-26]. The industrial manufacturers' benefits, using $\mathrm{mTG}$ were reported extensively. In brief, the $\mathrm{MTG}$ enzymatic action affects viscosity, gelation, foaming, thermal stability, elasticity, water-holding capacity, binding ability, emulsification, consistency, texture, resilience and above all, elongate the life time in the groceries, public markets and supermarkets shelves and improve palatability $[6,13,15]$. The enzyme is considered as an industrial processing aid, thus, escaping the regulation of a food additive.

\section{Celiac Disease and Tissue Transglutaminase}

Celiac disease (CD) is a gluten dependent autoimmune disease elicited in genetically predisposed individuals by the consumption of prolamine grains (i.e., wheat, barley, rye and oat) or ingredients of them. It affects $1-1.5 \%$ of Western populations and improves on gluten free diet. Pathophysiologically, gluten is partially digested in the enteric lumen, resulting in partial degradation till proteolytic-resistant peptides are formed. After trans-epithelial transport, gliadin peptides are deamidated/ transamidated by the sub-epithelial tTG, leading to the formation of deamidated gliadin peptides, have a stronger binding capacity to MHC II, thus, stimulating the $\mathrm{T}$ and $\mathrm{B}$ cells to damage the epithelium, induce inflammation and secrete $\mathrm{CD}$ associated autoantibodies [27]. It should be emphasized that often forgotten is that transamidation occurs at a higher rate $(75 \%)$ than deamidation (25\%) in the tTG-gliadin cross talks [28]. After transamidation, tTG is covalently linked to gliadin peptides to create neo-epitopes complexes [29]. Interestingly, these neo-epitopes were described in vivo in small intestine biopsies of $\mathrm{CD}$ patients where a 
pathogenic role was attributed to them [30-32]. Formation of tTG-neo epitopes and presentation to the immune system, would support the hypothesis of epitope spreading and the ensuing development of autoantibodies against tTG and against the neo-epitope tTG cross linked complex $[13,30,33]$. In summary, tTG is a key player in CD initiation and progression by modifying naive gliadins to immunogenic molecules and complexes, thus losing the tolerance to gluten/gliadin containing nutrients. The transamidation capacities of the tTG is imitated by the exogenous mTG.

\section{MICROBIAL TRANSGLUTAMINASE-GLIADIN CROSS-LINKED COMPLEXES ARE IMMUNOGENIC IN CELIAC DISEASE}

Several potential aspects associate mTG to celiac disease. At present, it should be stressed that we are dealing with an associative correlation and no causality was yet determined. Epidemiologically, the annual increased consumption of the enzyme goes parallel with the increased incidence of autoimmune conditions and $\mathrm{CD}$, in the last decades [14,34,35]. Pathophysiologically, mTG imitates functionally the tTG, both posttranslation modifiers of gluten/gliadin peptides, by deamidation and transamidation [6]. Chemically, gluten and gliadin peptides are ideal substrates for the two enzymes due to their rich glutamine and lower lysine contents. Sequence-wise, no sequence homology but active site similarity were detected upon alignment of the two TGs [13].

Those associations were at the basis to explore the immunogenicity of the mTG and its gliadin-docked complexes, in CD patients. When the serological titers of mTG, tTG, gliadin complexed mTG (mTG neo-epitope) and gliadin complexed tTG (tTG neo-epitope) were studied in 95 pediatric celiac patients, compared to 99 normal children, 79 normal adults and 45 children with nonspecific abdominal pain, the following results were obtained: (1) mTG-neo IgA, IgG and IgA combined with IgG antibody titers exceed significantly the comparable mTG ones. The anti mTG positive patients were negligible and with a very low activity. (2) All levels of mTG-neo and tTG-neo isotypes were significantly higher in CD patients compared to controls. (3) Comparing all studied antibodies, tTG-neo IgA+IgG, tTG-neo IgA and mTG-neo IgG correlated best with patient's intestinal pathology $\left(r^{2}=0.6454, r^{2}=0.6165, r^{2}=0.5633 ; p<0.0001\right.$, $p<0.0001, p<0.0001$, respectively). (4) mTG-neo IgG+IgA showed an increased immunoreactivity compared to single $\mathrm{mTG}$ and gliadin $(p<0.001)$ but similar immunoreactivity to the tTG-neo IgG and IgA ELISA. (5) Using competition ELISA, the MTG neo-epitopes and tTG neo-epitopes antibodies had identical outcomes when checked on CD sera, both showing a decrease in optical density of $55 \pm 6 \%$ ( $p<0.0002)$. The author's summary was: "mTG is immunogenic in children with $\mathrm{CD}$ and, by complexing to gliadin, its immunogenicity is enhanced" [13].

Comparing $17 \mathrm{CD}$ associated serological biomarkers, mTG-neo IgG correlated closely to the mucosal injury and was summarized as a new 
reliable serological biomarker for $\mathrm{CD}$ diagnosis and enteric damage reflection [27]. Finally, when a Swedish pediatric CD population was studied for CD relayed antibodies, mTG-neo IgG had a good area under curve on ROC analysis (0.877) and an acceptable sensitivity (0.88\%) and specificity (90\%) for CD diagnosis [36].

\section{PATHOGENIC ASPECTS OF MICROBIAL TRANSGLUTAMINASE}

The potential pathogenic proofs for the environmental mTG involvement in chronic human disease induction are still being explored, but some of them were already published. Figure 1 summarizes schematically the various pathogenic pathways of mTG, representing potential mechanisms for mTG pathogenicity.

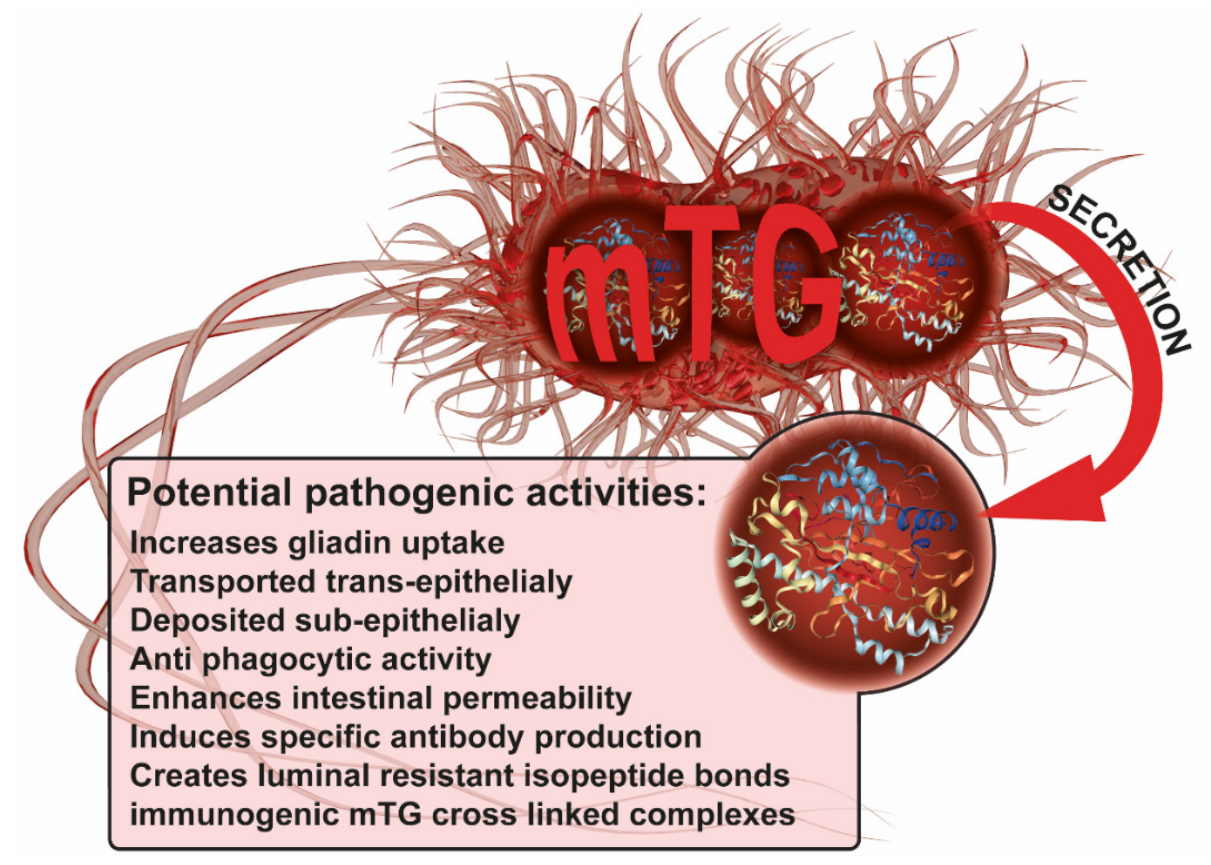

Figure 1. mTG activities representing potential mechanisms for mTG pathogenicity in human.

\section{mTG can Potentially Enhance Intestinal Permeability}

mTG is a survival factor that protects bugs against us, thus, enhancing the survival of dysbiotic or pathogenic bacteria in the gut lumen [2,3]. Microbial infections are a well-described etiology for compromising tight junction integrity and increasing intestinal permeability [37]. Gluten ingestion and gliadin peptides induce increased intestinal permeability, not only in CD patients [38]. Being a gluten-based peptide, mTG cross-linked gliadin can duplicate the effect on the tight junction. Additionally, actin, e-cadherin and adherens junctions are integral part of the enteric, inter-epithelial permeability machinery. They can be modified by TGs, including mTG that imitate functionally TGs, thus perturbing their protective ability [6,14,39]. mTG has emulsifying properties by cross-linking different proteins. Emulsifiers, heavily used in the process food industries, are enhancers of gut permeability $[14,40]$. 
The same reasoning apply to the mTG capacity to lipidate proteins, thus augmenting their emulsifying ability [41]. Much more, proteins originate from nutrients like casein, pork myofibrils, peanut and fish, cross-linked by mTG acquired emulsifying properties [39,42]. The same holds true for hydrolyzed gluten, known to increase emulsification [43]. Other food additive, heavily consumed by food industries, the nanoparticles, can be cross linked by mTG to improve their luminal delivery systems [44,45]. Nanoparticles also are disruptors of tight junction integrity [14].

Finally, Glutamine and sulfur-containing amino acids (cystine, cysteine and methionine) regulate the intestinal originated cell line (Caco-2) tight junction proteins. mTGs cross-linking those glutamine/sulfur containing amino acid peptides can induce a deprivation state of those amino acids, thus declining intestinal permeability regulation [46,47].

\section{mTG Effects on Epithelial Gliadin Uptake and Transportation}

tTG facilitates apical-basal passage of gliadins, a process helped by apical transferrin receptors and secretory IgA [48]. Imitating tTG functions, mTG potentially can facilitate this epithelial gliadin uptake pathway, thus enhancing CD [6]. Very recently, Stricker P. et al., shed a new aspect of MTG pathogenic potential [32]. They followed tagged mTG and gliadin, applied to CD intestinal biopsies and RACE cells (rapid uptake of antigen into the cytosol of enterocytes), ex vivo and in vitro, respectively. MTG and gliadin were transported to the enterocytes' and to the of RACE cells' endoplasmic reticulum. Furthermore, mTG strongly localized at the basolateral membrane and the enteric lamina propria. Those interesting observations suggest cross presentation of exogenous antigens, like mTG and gliadins, in CD patients and more importantly, indicating a potential antigenic interaction with cells of the immune system. The cited basic science study support our clinical studies of mTG-gliadin neo-epitope complexes, being immunogenic in CD patients [13,27]. Actually, the mechanism of those antibodies production is clearer, since foreign antigens like mTG and gliadin peptides, find their way from the intestinal lumen and after trans-enterocyte transport, deposited and exposed to the immune sub-epithelial system.

\section{mTG Suppresses Intestinal Luminal Protective Barriers}

Being exposed to the environment, the intestinal compartment evolved multiple protective mechanisms. Some of them can be disrupted by mTG, thus giving the bugs a survival advantage on us, in the enteric extremely hostile compartment.

A novel mTG was lately described in Streptococcus suis that represent a virulent factor that suppresses phagocytic activities, thus suppressing a crucial component of human immunity [49-51]. Not less significant is the mucus layer. Recently tTG was found to stabilize, by cross-linking glutamine rich compounds, the MUC2 Mucin intra cellular isopeptide bonds, before being secreted extracellularly [52]. Since the human gut 
lumen is rich in mTG activity, and the mucus is rich in acyl donors and acceptors, it is foreseeable that mTG can alter mucus stability, enabling pathogenic microbes to approach their attached receptors. Finally, the isopeptide bonds formed by mTG are extremely resistant to any human luminal enzyme, escaping the enzymatic degradation, reducing agents and multiple detergents. Even immunoglobulins, bile acids or antimicrobial molecules cannot break down those bonds [3,53,54]. In summary, luminal mTG has the capacity to counteract highly conserved evolutionary protective intestinal mechanisms.

\section{Additional Observations Related to Potential mTG Pathogenicity in CD}

One of the main important aspect is the availability of mTG in commercial food products. When 60 meat and meat products on the supermarket shelves were double-checked by different sensitive analytical methods, many of them contained mTG [55]. Although only associative, epidemiological survey showed a correlation between the increased $\mathrm{CD}$ incidence and the continuous surge in consumption of enzymes in the bakery industries, mTG being a major one $[6,14]$. Finally, wheat or gluten containing products enzymatically treated by mTG were shown to be immunogenic, inducing antibodies when consumed by humans [56-65], thus substantiating the serological studies on mTG immunogenicity [13,27].

\section{THE POTENTIAL INTESTINAL SOURCES OF MTG LOAD}

In order to exert its immunogenic and pathogenic activities (Figure 1), mTG needs to enter the human gut lumen. A portion of mTG is environmentally generated, some of it is introduced in the form of food additives and the rest is produced by the luminal prokaryotes [2,3]. Table 1 summarizes the extra and intra intestinal sources of mTGs. Taken together, a plethora of extra intestinal and enteric luminal sources of mTGs, capable to cross-link numerous substrates, including the glutamine rich gliadins, exist. The final result is that the mTG mobilomic cargo can postrtanslate and modify proteins, rendering naive to non-tolerated ones, potentially driving autoimmunity [2,3,6,32].

Table 1. The extra and intra-intestinal sources of mTG.

\begin{tabular}{lll}
\hline & mTG Source & References \\
\hline Extra-intestinal & Processed food additive & {$[2,3,6,8,13-17,24]$} \\
& Pathobionts & {$[2,3,14,50,66-68]$} \\
& Probiotics & {$[67,69-71]$} \\
& Plants & {$[65,72]$} \\
& Vegetables & {$[65,72]$} \\
Intra-intestinal & Microbiome & {$[2,3,6,13-17]$} \\
& Dysbiome & {$[2,3,6]$} \\
& Yeasts & {$[73-76]$} \\
\hline
\end{tabular}




\section{SUMMARY}

mTGs are considered, at least by producers, to be safe, non-toxic, non-allergenic, non-immunogenic and non-pathogenic for public health [6]. The present review summarizes the epidemiological, scientific and clinical proofs for this food additive and bacterial survival factor's immunogenic and pathogenic potentials. Actually, there is enough background knowledge to address mTGs' safety in a multi-disciplinary approach, aiming to protect the public against its potential detrimental effects. If substantiated, the findings will affect food product labeling, processed food additive policies, regulatory authorities' product control, consumer health education and public health safety. A.L. designed and wrote the manuscript, T.M. overviewed, searched and analyzed the literature and edited the manuscript.

\section{AUTHOR CONTRIBUTIONS}

A.L. conceived the original idea, designed and wrote the manuscript, T.M. overviewed, searched and analyzed the literature and edited the manuscript.

\section{CONFLICTS OF INTEREST}

No grant support and no conflicting interests.

\section{ACKNOWLEDGMENTS}

The authors would like to thank Neu Alf for the figure design and to Wusterhausen Patricia and Ramesh Ajay for the editing and reviewing of the manuscript.

\section{REFERENCES}

1. Lerner A, Neidhöfer S, Matthias T. Transglutaminase 2 and anti transglutaminase 2 autoantibodies in celiac disease and beyond: Part A: TG2 double-edged sword: gut and extraintestinal involvement. Immunome Res. 2015;11:101-5.

2. Lerner A, Aminov R, Matthias T. Dysbiosis may trigger autoimmune diseases via inappropriate posttranslational modification of host proteins. Front Microbiol. 2016;7:84.

3. Lerner A, Aminov R, Matthias T. Intestinal dysbiotic transglutaminases are potential environmental drivers of systemic autoimmunogenesis. Front Microbiol. 2017;8:66.

4. Katt WP, Antonyak MA, Cerione RA. The diamond anniversary of tissue transglutaminase: a protein of many talents. Drug Discov Today. 2018;23:575-91.

5. Agnihotri N, Mehta K. Transglutaminase-2: evolution from pedestrian protein to a promising therapeutic target. Amino Acids. 2017;49:425-39.

6. Lerner A, Matthias T. Possible association between celiac disease and bacterial transglutaminase in food processing: a hypothesis. Nutr Rev. 2015;73:544-52. 
7. Ando H, Adachi M, Umeda K, Matsuura A, Nonaka M, Uchio R, et al. Purification and characteristics of a novel transglutaminase derived from microorganisms. Agric Biol Chem. 1989;53:2613-7.

8. Martins IM, Matos M, Costa R, Silva F, Pascoal A, Estevinho LM, et al. Transglutaminases: recent achievements and new sources. Appl Microbiol Biotechnol. 2014;98:6957-64.

9. Strop P. Versatility of microbial transglutaminase. Bioconjug Chem. 2014;25:855-62.

10. Deweid L, Neureiter L, Englert S, Schneider H, Deweid J, Yanakieva D, et al. Directed Evolution of a Bond-Forming Enzyme: Ultrahigh-Throughput Screening of Microbial Transglutaminase Using Yeast Surface Display. Chemistry. 2018;24:15195-200.

11. Yu J, Pian Y, Ge J, Guo J, Zheng Y, Jiang H, et al. Functional and Structural Characterization of the Antiphagocytic Properties of a Novel Transglutaminase from Streptococcus suis. J Biol Chem. 2015;290:19081-92.

12. Reif S, Lerner A. Tissue transglutaminase-the key player in celiac disease: a review. Autoimmun Rev. 2004;3:40-5.

13. Matthias T, Jeremias $P$, Neidhöfer $S$, Lerner A. The industrial food additive microbial transglutaminase, mimics the tissue transglutaminase and is immunogenic in celiac disease patients. Autoimmun Rev. 2016;15:1111-9.

14. Lerner A, Matthias T. Changes in intestinal tight junction permeability associated with industrial food additives explain the rising incidence of autoimmune disease. Autoimmun Rev. 2015;14:479-89.

15. Kieliszek M, Misiewicz A. Microbial transglutaminase and its application in the food industry. A review. Folia Microbiol (Praha). 2014;59:241-50.

16. Rachel NM, Pelletier JN. Biotechnological applications of transglutaminases. Biomolecules. 2013;3:870-88.

17. Gaspar AL, de Góes-Favoni SP. Action of microbial transglutaminase (MTGase) in the modification of food proteins: a review. Food Chem. 2015;171:315-22.

18. Yokoyama K, Nio N, Kikuchi Y. Properties and applications of microbial transglutaminase. Appl Microbiol Biotechnol. 2004;64:447-54.

19. Mariniello L, Porta R, Sorrentino A, Giosafatto CV, Rossi Marquez G, Esposito M, Di Pierro P. Transglutaminase-mediated macromolecular assembly: production of conjugates for food and pharmaceutical applications. Amino Acids. 2014;46:767-76.

20. Fatima SW, Khare SK. Current insight and futuristic vistas of microbial transglutaminase in nutraceutical industry. Microbiol Res. 2018;215:7-14.

21. Deweid L, Avrutina O, Kolmar H. Microbial transglutaminase for biotechnological and biomedical engineering. Biol Chem. 2018 Oct 6. doi: 10.1515/hsz-2018-0335 [Epub ahead of print]

22. Zhang D, Zhu Y, Chen J. Microbial transglutaminase production: understanding the mechanism. Biotechnol Genet Eng Rev. 2010;26:205-22.

23. Malandain $\mathrm{H}$. Transglutaminases: a meeting point for wheat allergy, celiac disease, and food safety Europ. Ann. Aller. Clin. Immun. 2005;37:397-403. 
24. Santhi D, Kalaikannan A, Malairaj P, Arun Prabhu S. Application of microbial transglutaminase in meat foods: A review. Crit Rev Food Sci Nutr. 2017;57:2071-76.

25. Gharibzahedi SMT, Yousefi S, Chronakis IS. Microbial transglutaminase in noodle and pasta processing. Crit Rev Food Sci Nutr. 2017 Aug 31:1-15. doi: 10.1080/10408398.2017.1367643 [Epub ahead of print]

26. Taghi Gharibzahedi SM, Koubaa M, Barba FJ, Greiner R, George S, Roohinejad S. Recent advances in the application of microbial transglutaminase crosslinking in cheese and ice cream products: A review. Int J Biol Macromol. 2018;107(Pt B):2364-2374.

27. Lerner A, Jeremias P, Neidhöfer S, Matthias T. Comparison of the reliability of 17 celiac disease associated bio-markers to reflect intestinal damage. J Clin Cell Immunol. 2017;8:1.

28. Bizzaro N, Tozzoli R, Villalta D, Fabris M, Tonutti E. Cutting-Edge Issues in Celiac Disease and in Gluten Intolerance. Clin Rev Allergy Immunol. 2012;42:279-87.

29. Lerner A, Jeremias P, Neidhöfer S, Matthias T. Antibodies against neo-epitope tTg complexed to gliadin are different and more reliable then anti-tTg for the diagnosis of pediatric celiac disease. J Immunol Methods. 2016;429:15-20.

30. Porcelli B, Ferretti F, Vindigni C, Terzuoli L. Assessment of a Test for the Screening and Diagnosis of Celiac Disease. J Clin Lab Anal. 2016;30:65-70.

31. Ciccocioppo R, Di Sabatino A, Ara C, Biagi F, Perilli M, Amicosante G, et al. Gliadin and tissue transglutaminase complexes in normal and celiac duodenal mucosa. Clin Exp Immunol. 2003;134:516-24.

32. Stricker S, de Laffolie J, Rudloff S, Komorowski L, Zimmer KP. Intracellular Localization of Microbial Transglutaminase and Its Influence on the Transport of Gliadin in Enterocytes. J Pediatr Gastroenterol Nutr. 2018 Oct 12. doi: 10.1097/MPG.0000000000002171 [Epub ahead of print]

33. Matthias T, Neidhöfer S, Pfeiffer S, Prager K, Reuter S, Gershwin ME. Novel trends in celiac disease. Cell Mol Immunol. 2011;8:121-5.

34. Lerner A, Jeremias P, Matthias T. The world incidence and prevalence of autoimmune diseases is increasing: A review. Internat J Celiac Disease. 2015;3:151-5.

35. Lerner A, Jeremias P, Matthias T. The world incidence of celiac disease is increasing: a review. Int J Recent Sci Res. 2015;7:5491-6.

36. Agardh D. Diabetes and Celiac Disease Unit, Department of Clinical Sciences, Lund University, Malmö, Sweden. Personal communication.

37. Fasano A. Zonolin and its regulation of intestinal barrier function: the biological door to inflammation, autoimmunity, and cancer. Physiol Rev. 2011;91:151-75.

38. Hollon J, Puppa EL, Greenwald B, Goldberg E, Guerrerio A, Fasano A. Effect of gliadin on permeability of intestinal biopsy explants from celiac disease patients and patients with non-celiac gluten sensitivity. Nutrients. 2015;7:1565-76. 
39. Hiiragi T, Sasaki H, Nagafuchi A, Sabe H, Shen SC, Matsuki M, et al. Transglutaminase type 1 and its cross-linking activity are concentrated at adherens junctions in simple epithelial cells. J Biol Chem. 1999;274:34148-54.

40. Hu X, Ren J, Zhao M, Cui C, He P. Emulsifying properties of the transglutaminase treated crosslinked product between peanut protein and fish (Decapterus maruadsi) protein hydrolysates. J Sci Food Agric. 2011;91:578-85.

41. Abe H, Goto M, Kamiya N. Protein lipidation catalyzed by microbial transglutaminase. Chemistry. 2011;17:14004-8.

42. Hong GP, Min SG, Chin KB. Emulsion properties of pork myofibrillar protein in combination with microbial transglutaminase and calcium alginate under various pH conditions. Meat Sci. 2012;90:185-93.

43. Xiong YL, Agyare KK, Addo K. Hydrolyzed wheat gluten suppresses transglutaminase-mediated gelation but improves emulsification of pork myofibrillar protein. Meat Sci. 2008;80:535-44.

44. Fuchs S, Kutscher M, Hertel T, Winter G, Pietzsch M, Coester C. Transglutaminase: new insights into gelatin nanoparticle cross-linking. J Microencapsul. 2010;27:747-54.

45. Yew SE, Lim TJ, Lew LC, Bhat R, Mat-Easa A, Liong MT. Development of a probiotic delivery system from agrowastes, soy protein isolate, and microbial transglutaminase. J Food Sci. 2011;76:H108-15.

46. Li N, Neu J. Glutamine deprivation alters intestinal tight junctions via a PI3-K/Akt mediated pathway in Caco-2 cells. J Nutr. 2009;139:710-4.

47. Mullin JM, Skrovanek SM, Valenzano MC. Modification of tight junction structure and permeability by nutritional means. Ann N Y Acad Sci. 2009;1165:99-112.

48. Lebreton C, Menard S, Abed J, Moura IC, Coppo R, Dugave C, et al. Interactions among secretory immunoglobulin A, CD71, and transglutaminase-2 affect permeability of intestinal epithelial cells to gliadin peptides. Gastroenterology. 2012;143:698-707.

49. Fittipaldi N, Segura M, Grenier D, Gottschalk M. Virulence factors involved in the pathogenesis of the infection caused by the swine pathogen and zoonotic agent Streptococcus suis. Future Microbiol. 2012;7:259-79.

50. Xu B, Zhang P, Li W, Liu R, Tang J, Fan H. hsdS, Belonging to the Type I Restriction-Modification System, Contributes to the Streptococcus suis Serotype 2 Survival Ability in Phagocytes. Front Microbiol. 2017;8:1524.

51. Pian Y, Wang $\mathrm{P}$, Liu $\mathrm{P}$, Zheng $\mathrm{Y}$, Zhu L, Wang $\mathrm{H}$, et al. Proteomics identification of novel fibrinogen-binding proteins of Streptococcus suis contributing to antiphagocytosis. Front Cell Infect Microbiol. 2015;5:19.

52. Recktenwald CV, Hansson GC. The Reduction-insensitive Bonds of the MUC2 Mucin Are Isopeptide Bonds. J Biol Chem. 2016;291:13580-90.

53. Rao RU, Mehta K. Transglutaminases, thioredoxins and protein disulphide isomerase: diverse enzymes with a common goal of cross-linking proteins in lower organisms. Indian J Exp Biol. 2004;42:235-43.

54. Tagami U, Shimba N, Nakaamura M, Yokoyama K, Suzuki E, Hirokawa T. Substrate specificity of microbial transglutaminase as revealed by 
three-dimentional docking simulation and mutagenesis. Protein Eng Des Sel. 2009;22:747-52.

55. Kaufmann A, Koppel R, Widmer M. Determination of microbial transglutaminase in meat and meat products. Food Addit Contam Part A Chem Anal Control Expo Risk Assess. 2012;29:1364-73.

56. Lerner A, Matthias T. Food Industrial Microbial Transglutaminase in Celiac Disease: Treat or Trick. Int J Celiac Dis. 2015;3:1-6.

57. Cabrera-Chávez F, Rouzaud-Sández O, Sotelo-Cruz N, Calderón de la Barca AM. Bovine milk caseins and transglutaminase-treated cereal prolamines are differentially recognized by IgA of celiac disease patients according to age. J Agric Food Chem. 2009;57:3754-59.

58. Elli L, Roncoroni L, Hils M, Pasternack R, Barisani D, Terrani C, et al. Immunological effects of transglutaminase-treated gluten in coeliac disease. Hum Immunol. 2012;73:992-7.

59. Falini ML, Elli L, Caramanico R, Bardella MT, Terrani C, Roncoroni L, et al. Immunoreactivity of antibodies against transglutaminase-deamidated gliadins in adult celiac disease. Dig Dis Sci. 2008;53:2697-701.

60. Cabrera-Chávez F, Rouzaud-Sández O, Sotelo-Cruz N, Calderón de la Barca AM. Transglutaminase treatment of wheat and maize prolamines of bread increase the serum IgA reactivity of celiac disease patients. J Agric Food Chem. 2008;56:1387-91.

61. Berti C, Roncoroni L, Falini ML, Caramanico R, Dolfini E, Bardella MT, et al. Celiac-related properties of chemically and enzymatically modified gluten proteins. J Agric Food Chem. 2007;55:2482-8.

62. Heredia-Sandoval NG, Islas-Rubio AR, Cabrera-Chávez F, Calderón de la Barca AM. Transamidation of gluten proteins during the bread-making process of wheat flour to produce breads with less immunoreactive gluten. Food Funct. 2014;5:1813-8.

63. Ruh T, Ohsam J, Pasternack R, Yokoyama K, Kumazawa Y, Hils M. Microbial transglutaminase treatment in pasta-production does not affect the immunoreactivity of gliadin with celiac disease patients' sera. J Agric Food Chem. 2014;62:7604-11.

64. Dekking EHA, Van Veelen PA, de Ru A, Kooy-Winkelaara EMC, Gröneveld T, Nieuwenhuizen WF, Koning F. Microbial transglutaminase generate $\mathrm{T}$ cell stimulatory epitopes involved in celiac disease. J Cereal Sci. 2008;47:339-46.

65. Gerrard JA, Sutton KH. Addition of transglutaminase to cereal products may generate the epitope responsible for coeliac disease. Trends Food Sci Technol. 2008;16:510-2.

66. Skovbjerg H, Norén O, Anthonsen D, Moller J, Sjöström H. Gliadin is a good substrate of several transglutaminases: possible implication in the pathogenesis of coeliac disease. Scand J Gastroenterol. 2002;37:812-7.

67. Lerner A, Arleevskaya M, Schmiedl A, Matthias T. Microbes and viruses are bugging the gut in celiac disease. Are they friends or foes? Front Microbiol. 2017;8:1392.

68. Lerner A, Aminov R, Matthias T. Potential effects of horizontal gene exchange in the human gut. Front Immunol. 2017;8:1630. 
69. Devirgiliis C, Zinno P, Perozzi G. Update on antibiotic resistance in foodborne Lactobacillus and Lactococcus species. Front Microbiol. 2013;4:301.

70. Flórez AB, Campedelli I, Delgado S, Alegría Á, Salvetti E, Felis GE, et al. Antibiotic Susceptibility Profiles of Dairy Leuconostoc, Analysis of the Genetic Basis of Atypical Resistances and Transfer of Genes in vitro and in a Food Matrix. PLoS One. 2016;11:e0145203.

71. Rosander A, Connolly E, Roos S. Removal of antibiotic resistance gene-carrying plasmids from Lactobacillus reuteri ATCC 55730 and characterization of the resulting daughter strain, L. reuteri DSM 17938. Appl Environ Microbiol. 2008;74:6032-40.

72. Li C, Du G, Zhang D, Chen J. Thermal stability and conformational changes of transglutaminase from a newly isolated Streptomyces hygroscopicus. Bioresour Technol. 2008;99:3794-800.

73. Iranzo M, Aguado C, Pallotti C, Cañizares JV, Mormeneo S. Transglutaminase activity is involved in Saccharomyces cerevisiae wall construction. Microbiology. 2002;148:1329-34.

74. Ruiz-Herrera J, Iranzo M, Elorza MV, Sentandreu R, Mormeneo S. Involvement of transglutaminase in the formation of covalent cross-links in the cell wall of Candida albicans. Arch Microbiol. 1995;164:186-93.

75. Nieuwenhuizen WF, Pieters RH, Knippels LM, Jansen MC, Koppelman SJ. Is Candida albicans a trigger in the onset of coeliac disease? Lancet. 2003;361:2152-4.

76. Corouge M, Loridant S, Fradin C, Salleron J, Damiens S, Moragues MD, et al. Humoral immunity links Candida albicans infection and celiac disease. PLoS One. 2015;10:e0121776.

How to cite this article:

Lerner A, Matthias T. Microbial Transglutaminase is Beneficial to Food Industries but a Caveat to Public Health. Med One. 2019;4:e190001. https://doi.org/10.20900/mo.20190001 